\title{
Effects of prior hydrogenation on the structure and properties of thermally nanocrystallized silicon layers
}

\author{
A. Achiq, R. Rizk, ${ }^{\text {a) }}$ F. Gourbilleau, and R. Madelon \\ LERMAT, Unité CNRS 6004, ISMRA, 6 Bd. du Maréchal Juin, F-14050 Caen Cedex, France \\ B. Garrido, A. Pérez-Rodríguez, and J. R. Morante \\ Departament d'Electrònica, Universitat de Barcelona, Diagonal 645-647, 08028 Barcelona, Spain
}

(Received 30 September 1997; accepted for publication 26 February 1998)

\begin{abstract}
Nanocrystalline silicon layers have been obtained by thermal annealing of films sputtered in various hydrogen partial pressures. The as-deposited and crystallized films were investigated by infrared, Raman, x-ray diffraction, electron microscopy, and optical absorption techniques. The obtained data show evidence of a close correlation between the microstructure and properties of the processed material, and the hydrogen content in the as-grown deposit. The minimum stress deduced from Raman was found to correspond to the widest band gap and to a maximum hydrogen content in the basic unannealed sample. Such a structure relaxation seems to originate from the so-called "chemical annealing" thought to be due to $\mathrm{Si}-\mathrm{H}_{2}$ species, as identified by infrared spectroscopy. The variation of the band gap has been interpreted in terms of the changes in the band tails associated with the disorder which would be induced by stress. Finally, the layers originally deposited with the highest hydrogen pressure show a lowest stress-which does not correlate with the hydrogen content and the optical band gap-and some texturing. These features are likely related to the presence in these layers of a significant crystalline fraction already before annealing. (C) 1998 American Institute of Physics. [S0021-8979(98)00511-8]
\end{abstract}

\section{INTRODUCTION}

The microstructural features of polycrystalline semiconductors continue to raise great interest on account of their ability to govern the electrical and optical properties of these materials. ${ }^{1}$ Among them, the nanocrystalline silicon (nc-Si) thin films hold a distinguished place because of their potential for microelectronic devices such as solar cells ${ }^{2}$ and thin film transistors for flat panel displays. ${ }^{3}$

The most common way for the preparation of $n c-\mathrm{Si}$ films consists in the thermal crystallization of an hydrogenated amorphous silicon layer $(a-\mathrm{Si}: \mathrm{H})$. For all the chemical vapor deposition (CVD) techniques that are widely used for the fabrication of silicon thin films, hydrogen is always incorporated through the H-rich plasma or the gas vectors. On the contrary, sputtering technique allows the control of hydrogen incorporation in the deposits. Considering the key role ascribed to hydrogen in the crystallization mechanisms, the concentration and configurations of this element in the as-deposited layer are important to be determined for a better control of the crystallized silicon structure. In this connection, it is known that the high dilution of $\mathrm{SiH}_{4}$ or $\mathrm{Si}_{2} \mathrm{H}_{6}$ in $\mathrm{H}_{2}$ for CVD techniques, ${ }^{4,5}$ or the prominence of hydrogen in the sputtering plasma, ${ }^{6}$ lead in both cases, to the direct deposition of $n c$-Si thin films. This is due to a preferential etching during growth of energetically unfavorable bonds at the surface of the growing films by the hydrogen species. ${ }^{7}$ The prior incorporation of hydrogen may increase the nucleation sites and influence also the growth kinetics of the silicon crystal-

\footnotetext{
a) Author to whom correspondence should be addressed; electronic mail:
} richard.rizk@lermat.ismra.fr lites. Thus, the nature of the grain boundaries in the thermally crystallized silicon would depend on the hydrogen content and configurations in the basic material. Comparing the grain boundary region to an amorphous network, the stored energy in the strained and distorted bonds may vary by a factor of 2 depending on the degree of structural relaxation, in a similar way to that reported for the amorphous material. ${ }^{8}$ Within this context, it is of prime importance to examine the effects of prehydrogenation on the microstructure of the thermally crystallized Si films. The present work deals with detailed structural analyses of $n c$-Si layers obtained by thermal annealing of sputtered films containing various concentrations of hydrogen. The as-deposited and thermally nanocrystallized layers have been investigated by infrared (IR) spectroscopy, Raman scattering, x-ray diffraction (XRD), conventional and high resolution electron microscopy (CTEM and HREM) techniques, in combination with optical absorption measurements.

\section{EXPERIMENT}

The films used in this study were deposited by radiofrequence (rf) sputtering of a $10 \Omega \mathrm{cm}$ silicon target of $100 \mathrm{~mm}$ diameter. Quartz and single crystal (001) Si wafers were used as substrates. The total pressure, $P_{\text {tot }}$, of argon or a mixture of argon and hydrogen was kept constant at 2 $\times 10^{-3}$ Torr, while the ratio of hydrogen partial pressure $r_{\mathrm{H}}=P_{\mathrm{H}_{2}} / P_{\text {tot }}$ was varied from $0 \%$ to $75 \%$. During deposition, the rf power and substrate temperature were maintained at $0.3 \mathrm{~W} / \mathrm{cm}^{2}$ and $250{ }^{\circ} \mathrm{C}$, respectively. The thickness of the 

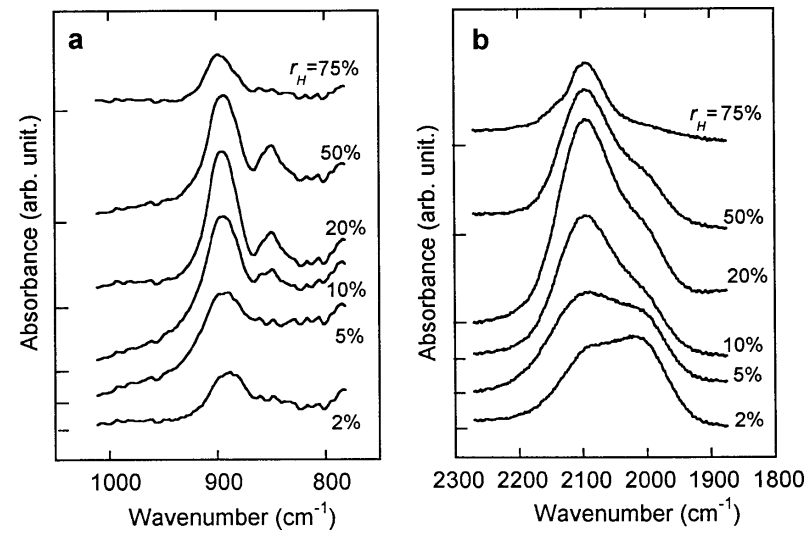

FIG. 1. Infrared spectra of as-deposited layers at the indicated hydrogen partial pressure ratios $r_{\mathrm{H}}$ in the regions $750-1050 \mathrm{~cm}^{-1}$ (a) and $1800-2300 \mathrm{~cm}^{-1}$ (b).

deposits was ranging from 0.9 to $1.4 \mu \mathrm{m}$. The concentration and configurations of hydrogen were determined by IR absorption measurements using a Fourier transform Nicolet 750-II spectrometer.

The layers were thermally crystallized at $720{ }^{\circ} \mathrm{C}$ during $90 \mathrm{~min}$ in a double wall quartz tube furnace under a continuous flow of pure nitrogen. To prevent the abrupt eruption of hydrogen, the films were gradually and slowly introduced in the furnace which was first heated at the annealing temperature. The crystalline fraction and the internal stress were estimated from the analyses of the Raman spectra. The Raman scattering measurements were performed in a Jobin-Yvon T6400 spectrometer coupled with an Olympus microscope. The measurements were done in backscattering configuration using an exciting wavelength of $514.5 \mathrm{~nm}$ from an $\mathrm{Ar}^{+}$laser. The spot was focused on a diameter of about $0.7 \mu \mathrm{m}$ on the sample surface and the excitation power density was kept below $0.26 \mathrm{MW} / \mathrm{cm}^{2}$ to avoid any heating effect.

The crystallite size and texture were determined by XRD, CTEM, and HREM techniques. For the former, the analyses were carried out by means of a high resolution Phillips instrument in the standard $\theta-2 \theta$ configuration. For electron microscopy observations, the samples were thinned and then glued with deposited films facing each other using the well known cross-section technique. While the CTEM investigations were made by means of a Jeol $200 \mathrm{CX}$ equipment operating at $200 \mathrm{kV}$, the HREM observations were performed in a Topcon 002B operating at $200 \mathrm{kV}$ allowing a resolution of $1.8 \AA$.

Finally, optical absorption measurements were performed between 0.4 and $4.1 \mathrm{eV}$ in a double-beam PerkinElmer spectrophotometer, allowing the determination of the optical band gap, $E_{\mathrm{og}}$, and the refractive index, $n$.

\section{RESULTS}

\section{A. Infrared measurements}

The IR measurements performed on the as-deposited samples aimed at the determination of both hydrogen content and hydrogen configurations before annealing. The IR spectra recorded on these samples and reported in Fig. 1, show

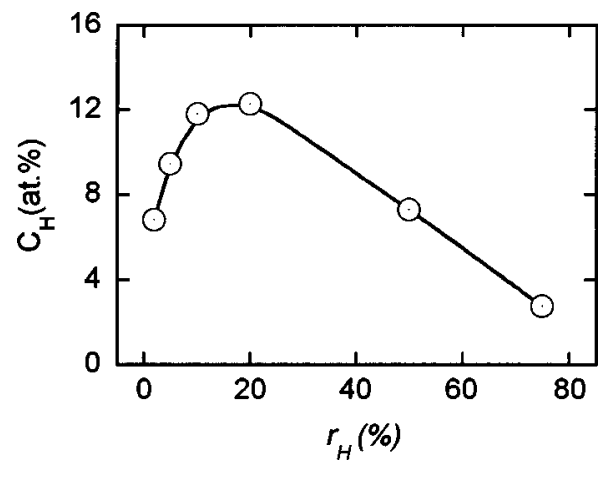

FIG. 2. Evolution of the bonded hydrogen content in the as-deposited films vs the ratio of the hydrogen partial pressure $r_{\mathrm{H}}$.

the $750-1050 \mathrm{~cm}^{-1}$ bending mode range (a) and the $1800-2300 \mathrm{~cm}^{-1}$ stretching mode range (b). The concentration of bonded hydrogen, $C_{\mathrm{H}}$, has been deduced from the integral over the wagging modes (not shown) that are located around $640 \mathrm{~cm}^{-1}$ for the various hydrid species, following the expression,

$$
C_{\mathrm{H}}=A_{640} \int \frac{\alpha(\omega)}{\omega} d \omega,
$$

where $\alpha$ is the absorption coefficient, $\omega$ is the frequency in $\mathrm{cm}^{-1}$, and $A_{640}$ is the proportionality constant taken equal to $2.1 \times 10^{19} \mathrm{~cm}^{-2}$, according to Langford et al. ${ }^{9}$ From the variation of $C_{\mathrm{H}}$ vs $r_{\mathrm{H}}$, shown in Fig. 2, we notice the gradual increase of the hydrogen concentration when $r_{\mathrm{H}}$ increases from $0 \%$ to $20 \%$, and then, its decrease when $r_{\mathrm{H}}$ is increased further $(\geqslant 50 \%)$. The various hydrogen configurations are usually differenciated by their stretching and/or bending modes. In this respect, the $2000 \mathrm{~cm}^{-1}$ vibration is attributed to isolated monohydrides $(\mathrm{Si}-\mathrm{H})$, while the 2090-2100 $\mathrm{cm}^{-1}$ modes can be ascribed to dihydrides $\left(\mathrm{Si}-\mathrm{H}_{2}\right)$ and/or clustered monohydride species. ${ }^{10-12}$ The detection of $\mathrm{Si}-\mathrm{H}_{2}$ bending vibrations and the modes of $\left(\mathrm{Si}-\mathrm{H}_{2}\right)_{n}$ chains at around 890 and $840 \mathrm{~cm}^{-1}$, respectively, ${ }_{13}$ confirms the formation and, therefore, the contribution of $\mathrm{Si}-\mathrm{H}_{2}$ in the spectra shown in Fig. 1(b).

More particularly, the increase of $C_{\mathrm{H}}$ in the $0 \%-20 \%$ interval of $r_{\mathrm{H}}$ values, is mainly due to the increase of $2100 \mathrm{~cm}^{-1}$ component, whereas the $2000 \mathrm{~cm}^{-1}$ band remains almost unchanged. In contrast, the gradual reduction of $C_{\mathrm{H}}$ for $r_{\mathrm{H}} \geqslant 50^{\circ} \mathrm{C}$ is originated from the decrease until extinction of the $2000 \mathrm{~cm}^{-1}$, together with the lowering of the $2100 \mathrm{~cm}^{-1}$ peak.

\section{B. Raman spectroscopy measurements}

The analyses of the Raman scattering measurements performed on the as-grown and the annealed layers have allowed the estimate of the crystalline fraction, the correlation length, $L$ (related to phonon confinement effects in the crystallite), and the stress, $\sigma$.

The Raman scattering spectra from amorphous silicon is characterized by the presence of broad bands centered at $480 \mathrm{~cm}^{-1}$ (TO), $380 \mathrm{~cm}^{-1}$ (LO), $310 \mathrm{~cm}^{-1}$ (LA), and $150 \mathrm{~cm}^{-1}$ (TA), while crystalline silicon only shows a first 


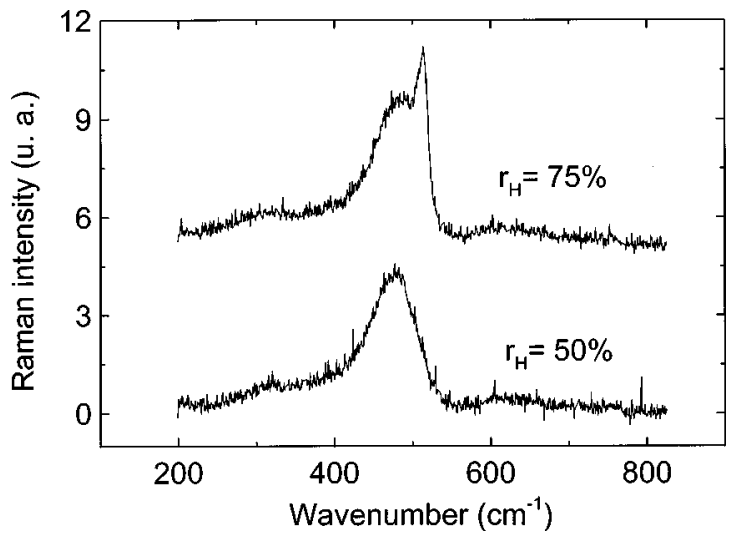

FIG. 3. Raman spestra of the as-deposited films at the indicated hydrogen partial pressure ratios $r_{\mathrm{H}}$.

order line with a lorentzian shape centered at $521 \mathrm{~cm}^{-1}$. This corresponds to a triply degenerated zone centered phonon.

The Raman spectra measured on the as-deposited films with $r_{\mathrm{H}} \leqslant 20 \%$ do not show any contribution from crystalline mode. This indicates that we are dealing with completely amorphous layers. In contrast, for $r_{\mathrm{H}} \geqslant 50 \%$, a crystalline contribution arises in the spectra shown in Fig. 3, which reflects the partial crystallization of the layers during deposition. This is maximum for sample deposited at $r_{\mathrm{H}}=75 \%$ which shows a very much higher crystalline contribution. Note that $C_{\mathrm{H}}$ increases with $r_{\mathrm{H}}$ as far as the deposit is amorphous, but it decreases when crystallites start to form (see Fig. 2).

The Raman scattering spectra recorded on the thermally crystallized silicon layers are compared in Fig. 4. The crystalline contribution in the spectra was obtained by fitting the amorphous bands and substracting this fitting from the experimental spectra. Moreover, we noticed some changes in the shape and position of the crystalline peak with respect to the corresponding ones for monocrystalline silicon. For these modifications, neither the Fano effects, nor the thermal effects can be taken into consideration. The reason lies for the former in the low doping of the target, and, for the latter, in the absence of any alteration upon changing the excitation power in the range of values used in this work. Accordingly,

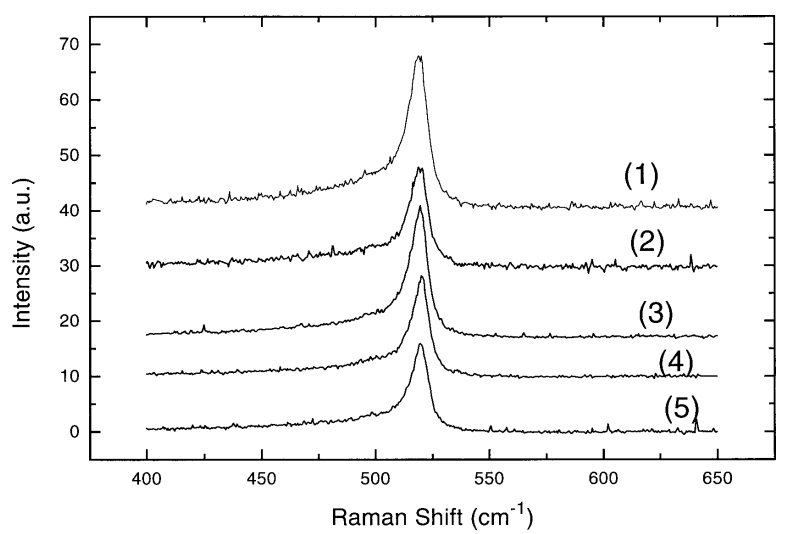

FIG. 4. Raman spectra of the thermally crystallized samples which have been deposited with different hydrogen partial pressure ratio $r_{\mathrm{H}}$ : (1) $0 \%$; (2) $10 \%$; (3) $20 \%$; (4) $50 \%$; (5) $75 \%$. the observed shifts and distortions are likely due to disorder effects related to the presence of structural effects and which confine the phonons and the stresses in the crystallites. Assuming that both disorder effects and stress are uniform in the scattering volume, the fitting of the spectra with the correlation length model (CLM) allows one to estimate both the correlation length, $L$, which is related to the average distance between defects, and the stress value, $\sigma .{ }^{14-17}$ Of course, both magnitudes have to be considered as effective average values as in principle some distribution of both $L$ and $\sigma$ might be present in the layers. On the basis of the loss of the translational symmetry in highly disordered materials, the CLM assumes a relaxation of the momentum conservation rule. The intensity of the first order Raman line is given by

$$
I(\omega) \propto \int_{0}^{2 \pi / a_{0}} \frac{|C(q)|^{2} 4 \pi q^{2} d q}{(\omega-\omega(q))^{2}+\left(\Gamma_{0} / 2\right)^{2}},
$$

where $q$ is the wave vector, $\Gamma_{0}$ is the Raman intrinsic linewidth of crystalline silicon, and $C(q)$ is an appropriate multiplying function that localizes the mode inside a finite space. For spherical shape, $C(q)$ is Gaussian:

$$
|C(q)|^{2} \propto \exp \left[-q^{2} L^{2} / 8\right] .
$$

The phonon dispersion relation $\omega(q)$ is taken according to Ref. 18:

$$
\omega(q)=\omega_{p}-120\left(q / q_{0}\right)^{2},
$$

where $\omega_{p}$ is the wave number of the first order Raman band in the absence of disorder effects and $q_{0}=2 \pi / a_{0}\left(a_{0}\right.$ : lattice constant).

Owing to phonon confinement, the correlation length would correspond to the average distance between defects which are breaking translational symmetry such as grain boundaries, precipitates, dislocations, and cavities. $L$ is then expected to be smaller than the grain size when the grain contain a high density of defects, but it would give an estimate of the grain size in the case of low amount of defects.

Although the shape of the crystalline-related line can be accurately fitted, the peak position is also affected by the presence of stress, $\sigma$, in the scattering volume. The stressinduced shift is defined as

$$
\Delta \omega_{s}=\omega_{p}-\omega_{0},
$$

where $\omega_{0}$ is the wave number of the first order Raman band in the absence of both disorder and stress effects $\left(521 \mathrm{~cm}^{-1}\right)$. Assuming an in-plane stress model: ${ }^{19}$

$$
\Delta \omega_{s}=-3.2 \sigma \mathrm{cm}^{-1} \mathrm{GPa}^{-1} .
$$

The values of $L$ and $\sigma$, reported in Table I, have been estimated for all the crystallized samples on the basis of the above-mentioned assumptions. These values suggest that $L$ is almost constant ranging from 65 to $75 \AA$, and that $\sigma$ is compressive. This latter shows a minimum for $r_{\mathrm{H}}=20 \%$ and its values correlate with the hydrogen content plotted in Fig. 2 , except for the sample deposited with the highest value of $r_{\mathrm{H}}(75 \%)$. This provides a clear indication of the achievement of an increasingly relaxed structure when the unannealed basic layer is increasingly hydrogenated. 
TABLE I. The correlation length $L$ and the stress $\sigma$ values, as deduced from the correlation length model describing the Raman scattering for the nanocrystallized samples that have been deposited before annealing at the indicated ratios of hydrogen partial pressure $r_{\mathrm{H}}$. Also reported are the values of the corresponding crystalline fraction $f_{c}$ and refractive index $n$ deduced from Raman and optical absorption measurements, respectively.

\begin{tabular}{rcccc}
\hline \hline$r_{\mathrm{H}}(\%)$ & $L(\AA)$ & $\sigma(\mathrm{MPa})$ & $f_{c}(\%)$ & $n$ \\
\hline 0 & 66 & -496.9 & 71 & 3.10 \\
2 & 70 & -960.4 & 70 & 3.18 \\
10 & 65 & -812.5 & 70 & 3.19 \\
20 & 72 & -681.3 & 70 & 3.25 \\
50 & 70 & -1040.6 & 75 & 3.29 \\
75 & 75 & -637.5 & 70 & 3.32 \\
\hline \hline
\end{tabular}

Table I reports also the crystalline fraction as deduced from the integrated intensity $I_{c}$ and $I_{a}$ of the crystalline and amorphous bands, respectively:

$$
f_{c}=\frac{I_{c}}{I_{c}+\gamma I_{a}},
$$

where $\gamma$ is the ratio of the Raman efficiencies of the crystalline and amorphous parts. Following Ref. 20, $\gamma$ can be determined from the expression

$$
\gamma(L)=0.1+\exp [-L(\AA) / 250] .
$$

The data of Table I have been obtained with $L=70 \AA$ as an average value for all samples. They indicate a crystallinity which appears almost independent of the prior hydrogen content.

\section{X-ray diffraction and electron microscopy}

Beside the usual absorption factor (thickness of the sample, irradiated area), the experimental XRD intensities obtained with our nanocrystallized films, have been corrected by standard intensities relating to a randomly oriented silicon powder. The normalized contributions of the (111), (220), and (311) diffraction peaks obtained on our samples are plotted in Fig. 5 as a function of $r_{\mathrm{H}}$. Up to $r_{\mathrm{H}} \leqslant 50 \%$, there is a randomly distributed orientation of the nanocrys-

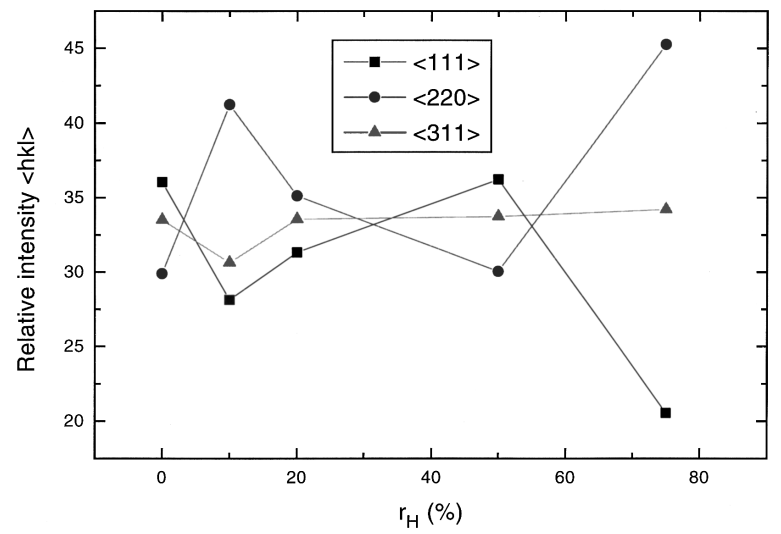

FIG. 5. Normalized intensities of the $\langle 111\rangle,\langle 220\rangle$, and $\langle 311\rangle \mathrm{x}$-ray reflections from the thermally crystallized layers, vs the hydrogen partial pressure ratio $r_{\mathrm{H}}$ of the basic deposits, i.e., before annealing. The contributions from higher angle reflections are negligible.

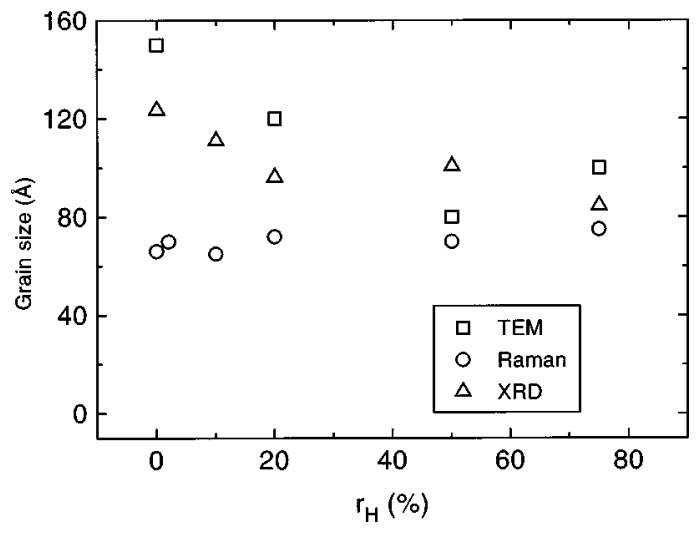

FIG. 6. Variations of the average grain size values obtained from TEM observations and from XRD measurements, compared to that of the correlation length values deduced from Raman measurements of the crystallized samples, as a function of the hydrogen partial pressure ratio $r_{\mathrm{H}}$ of the originally unannealed layers.

tals, whereas for $r_{\mathrm{H}}=75 \%$, there is a clear (220) preferential orientation at the expense of the (111) direction. On the other hand, the average grain size $D$ has been determined by XRD using the Scherrer formula:

$$
D=K \lambda / w \cos \theta
$$

where $K \sim 1, \lambda$ is the $\mathrm{x}$-ray wavelength, $w$ the width of the band, and $\theta$ the Bragg angle. The evolution of $D$ values with regard to $r_{\mathrm{H}}$ is shown in Fig. 6.

The electron microscopy observations realized on the annealed layers have corroborated their nanocrystalline structure and have enabled us to estimate the grain size in the range from 80 to $140 \mathrm{~nm}$. These values are compared in Fig. 6 to those deduced from XRD measurements and also to the Raman correlation lengths. The values obtained by XRD and CTEM are similar and show a trend to decrease when $r_{\mathrm{H}}$ increases, whereas the Raman correlation length appears almost constant, as emphasized above. It is worth noticing that for the highest value of $r_{\mathrm{H}}(75 \%)$, the XRD, CTEM, and $L$ values become all comparable. From the representative HREM image reported in Fig. 7, the grains appear mostly

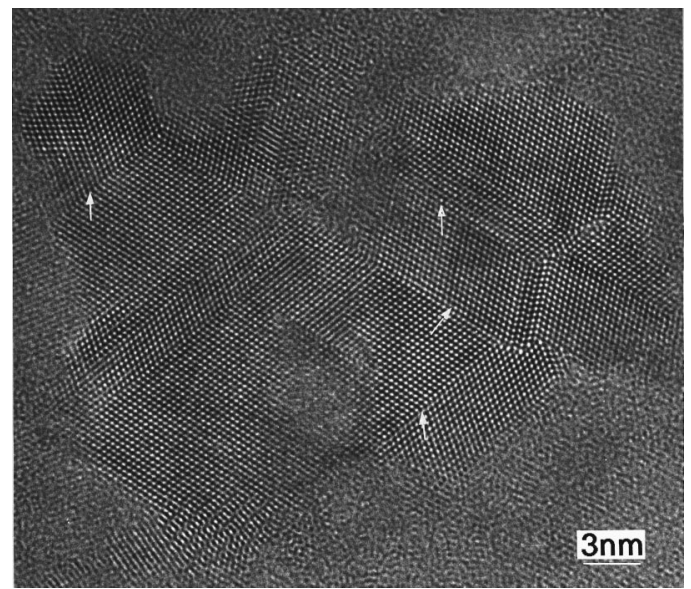

FIG. 7. High resolution electron microscopy image of the crystallized sample corresponding to a deposit with an hydrogen partial pressure ratio $r_{\mathrm{H}}$ of $20 \%$. The arrows indicate some microtwins present in the grains. 


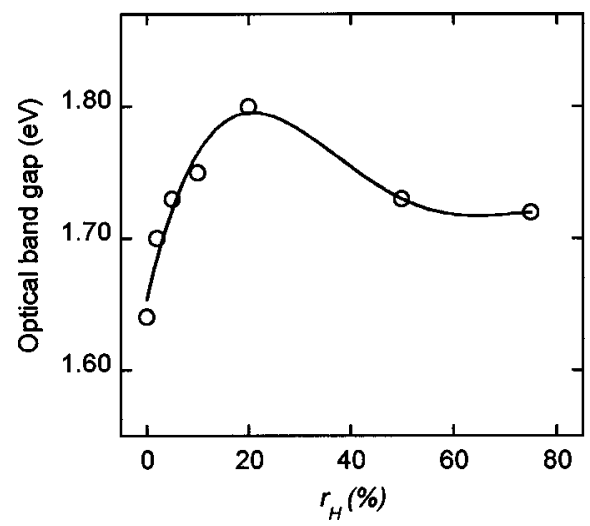

FIG. 8. Values of the optical band gap of the nanocrystallized samples versus the hydrogen partial pressure ratio $r_{\mathrm{H}}$ used for the deposition of the layers before annealing.

polygonal with (111) limiting surfaces, in addition to some steps and terraces. A careful observation of this image allows one to notice the existence of microtwins (some are indicated by arrows), whose separating distance to the nearest grain boundary is comparable to $L$.

\section{Optical absorption}

The above-described results related to the structural features have been complemented by optical absorption measurements. Figure 8 shows the behavior of the optical gap $E_{\text {og }}$ for the various samples, as deduced from the Tauc plots: $^{21}$

$$
(\alpha h \nu)^{1 / 2} \propto\left(h \nu-E_{\mathrm{og}}\right),
$$

where $h \nu$ is the photon energy and $\alpha$ is the absorption coefficient. $E_{\text {og }}$ shows an evolution that is, on the one hand, strikingly similar to that of $C_{\mathrm{H}}$ and, on the other hand, systematically opposite to the variation of the compressive stress $\sigma$ (see Table I). Otherwise, the refractive index measured at $1.9 \mu \mathrm{m}$, gives values which are indicative of a slightly increasing compactness with $r_{\mathrm{H}}$ (see Table I).

\section{DISCUSSION}

To examine the hydrogen-related features in the samples before annealing, we will comment first their IR spectra shown in Fig. 1. These latter indicate a $\mathrm{Si}-\mathrm{H}_{2}$ content in the amorphous films (for $r_{\mathrm{H}}<50 \%$ ) higher than that of the monohydride bonds. This seems characteristic of sputtered amorphous silicon, ${ }^{22}$ except the case of $r_{\mathrm{H}}=2 \%$ which shows a higher contribution of monohydride bonds $\left(2000 \mathrm{~cm}^{-1}\right)$. The formation of these $\mathrm{Si}-\mathrm{H}$ bonds would be favored by the low hydrogenation rate $\left(r_{\mathrm{H}}=2 \%\right)$, together with the deposition at a moderate $\left(250{ }^{\circ} \mathrm{C}\right)$ substrate temperature. $^{23}$

On the other hand, Fig. 2 indicates that the hydrogen concentration, $C_{\mathrm{H}}$, increases with $r_{\mathrm{H}}$ as far as the asdeposited layer is amorphous $\left(0 \% \leqslant r_{\mathrm{H}} \leqslant 20 \%\right)$, but it decreases for higher values of $r_{\mathrm{H}}(>50 \%)$ where some degree of crystallization starts to take place. Such a partial crystallization is in accordance with previous reports dealing with similar predominance of hydrogen partial pressure in the sputtering plasma. ${ }^{24,25}$ The desorption of hydrogen accompanying the direct crystallization has been already noticed ${ }^{6,26,27}$ and is generally ascribed to complex interactions between the silicon hydrides $\left(\mathrm{Si}-\mathrm{H}_{2}, \mathrm{Si}-\mathrm{H}_{3}\right)$ produced by the reactive sputtering of the silicon target by hydrogen, on the one side, and the weak or strained bonds at the growing surfaces, on the other side. This is known to lead to the crystalline state, together with the formation of volatile species such as $\mathrm{SiH}_{4}$, $\mathrm{Si}_{2} \mathrm{H}_{6}$, and $\mathrm{H}_{2}$.

Turning now to the thermally nanocrystallized samples, the data provided by Raman measurements allow one to note the compressive character of the stress $\sigma$ whose values of some hundreds of MPa remain significantly lower than those of 2000-4000 of MPa reported by Iqbal et al. for samples obtained by chemical transport method using a hydrogen plasma. $^{28}$ This allows to point out the relatively relaxed structure of our material. Besides, the $\sigma$ values of Table I show a net decrease when $C_{\mathrm{H}}$ is increased for the values of $r_{\mathrm{H}}$ below $50 \%$ (Fig. 2). To attempt an explanation of this aspect, we recall that hydrogen is usually associated with a more relaxed structure since it releases the distorted bonds and would lead to the formation of some microvoids. Thus, upon solid phase crystallization, and particularly for a gradual and moderate heating as in our case, the existing hydrogen is expected to contribute to the relaxation of the crystallized structure. This would be increasingly more effective for increasingly higher concentration of hydrogen $\left(C_{\mathrm{H}}\right)$ in the basic material. In this respect, we note that the major contributor to $C_{\mathrm{H}}$ is $\mathrm{SiH}_{2}$ species. Because of their highly unstable and reactive character, ${ }^{29}$ these species might contribute, by the so-called "chemical annealing," (Ref. 7), to both crystallization and relaxation during the abovementioned smooth annealing. This contribution consists in the breaking of strained and weak $\mathrm{Si}-\mathrm{Si}$ and $\mathrm{Si}-\mathrm{H}$ bonds, leading to much less distorted bonds upon thermal crystallization. Using nuclear magnetic resonance technique, the role of $\mathrm{Si}-\mathrm{H}_{2}$ in the improvement of the microcrystalline silicon structure has been recently clearly evidenced. ${ }^{30}$ On the other hand, samples deposited with high values of $r_{\mathrm{H}}$ are found of composite character (nanograins grown in amorphous tissue). This takes place especially for the sample deposited with $r_{\mathrm{H}}=75 \%$, which accounts for the deviation of the measured stress from the behavior observed for the other samples. As plotted in Fig. 2, the samples deposited with $r_{\mathrm{H}} \geqslant 50 \%$ show smaller $C_{\mathrm{H}}$ and therefore more compactness, as corroborated by the refractive index values reported in Table I.

Concerning the correlation length estimated from Raman scattering measurements, the comparison of its values ranging between 66 and $75 \AA$ to those of the grain size deduced from XRD measurements and CTEM observations (see Fig. 6 ), allows one to stress on the smaller values of the former. As emphasized above, $L$ represents the average distance between defects and it is always found lower than the grain size determined by other techniques. This is confirmed by previous results reported for microcrystalline silicon obtained by molecular beam epitaxy. ${ }^{31}$ There, beside a grain size of hundreds of $\AA$, as determined by CTEM, $L$ was found lower than $150 \AA$ and has been considered as the limiting factor for the 
conductivity. ${ }^{31}$ This demonstrates the relevance of our approach in combining the Raman scattering to other structural characterization techniques such as CTEM and XRD. Consequently, our $L$ values imply the existence of defects in our crystals such as the microtwins evidenced by the HREM image of Fig. 7. The average distance between these microtwins (some are indicated by arrows) or that separating these defects to the nearest grain boundary are both comparable to $L$. The almost constant value of this latter with $r_{\mathrm{H}}$ suggests a similar density of structural defects in the grains from the different samples. For high values of $r_{\mathrm{H}}$, the grain size provided by CTEM and XRD decreases and approaches the correlation length $L$. This suggests that for these small crystals $(\sim 80 \AA)$, the grain size becomes comparable to the average distance between defects (given by $L$ ), pointing out the low amount of defects in these grains, which is relevant for the electronic properties of the material.

It is worth noticing, however, that neither the grain size values ranging between 80 and $140 \AA$, nor their variation with $r_{\mathrm{H}}$, are able to give an account of the magnitude and, more particularly, of the evolution of the optical band gap $E_{\text {og }}$ shown in Fig. 8. The eventual influence of microvoid density on the behavior of $E_{\mathrm{og}}$ can be excluded since the values of the refractive index reported in Table I are rather indicative of an increasing compactness with $r_{\mathrm{H}}$. On the contrary, it is important to point out the striking resemblance between the evolution of $E_{\mathrm{og}}$ and $C_{\mathrm{H}}$ (Figs. 2 and 8), on the one hand, and, the opposite behavior of the stress $\sigma$ (Table I) versus $r_{\mathrm{H}}$, on the other hand. Considering the band tails of localized states that can be induced by the disorder ${ }^{32}$ and the stress in our material, we can argue that the most relaxed structure, where the stress is minimum $\left(r_{\mathrm{H}}=20 \%\right)$, would correspond to the narrower band tail and, consequently, to the widest band gap. This explanation is strongly corroborated by the evolution of the Urbach energy, $E_{u}$, measured on the same samples and reported elsewhere. ${ }^{33}$ The Urbach parameter, $E_{u},{ }^{34}$ which corresponds to the above-mentioned band tail and is associated with the topological disorder ${ }^{32}$ including structural, chemical, and thermal disorder, has been found minimum when the as-grown unannealed film was the most highly hydrogenated $\left(r_{\mathrm{H}}=20 \%\right)$. In contrast, the sample deposited with $r_{\mathrm{H}}=75 \%$ has already a significant amount of nanocrystallites (Fig. 3) before annealing, and has the lowest hydrogen content. The presence of these nanocrystals involves an inhomogeneous distribution of nucleation sites which would generate, upon annealing, interpenetrating areas of various degrees of disorder. This could explain the differences observed in the stress and texture data in relation to the other samples which, in any case, reflect the different recrystallization mechanisms. Hence, in general the variation of $E_{\mathrm{og}}$ is likely determined by the variation of the extent of the band tails, i.e., $E_{u}$. These suggestions are supported by the observations reported in a recent study dealing with identically heat treated $\mathrm{Si}$ samples after similar deposition by sputtering but from $\mathrm{H}$-free plasma. ${ }^{35}$ For the same annealing temperature, the optical band gap determined by these authors $(\sim 1.7 \mathrm{eV})$ is close to ours, and they ascribe the widening of $E_{\text {og }}$ to the strain relaxation produced by the prolonged annealing. Thus, it seems that the increase of the hydrogen content in the basic material (i.e. before annealing) would give similar effects to those determined by the annealing duration at a given temperature. In both cases, we notice a release of the stress field, then the narrowing of the band tails which, in turn, leads to the observed widening of $E_{\mathrm{og}}$.

Regarding texture, the random distribution of the grain orientation noticed for $r_{\mathrm{H}} \leqslant 50 \%$, (Fig. 5) could result from the great multiplicity of the nucleation sites and/or from the homogeneously distributed imperfections in the as-deposited layers where nucleation occurs predominantly. ${ }^{36}$ The former feature may originate, as already observed for $\mathrm{H}_{2}$-diluted plasma-enhanced CVD ${ }^{37}$ from the incorporation of hydrogen in the deposit, while the latter could be due to the amorphous structure of the unannealed samples where defects are expected to be evenly spread. In all these cases, the growth of crystallites would occur on a randomly oriented nuclei with the assistance of mobile hydrogen species. However, the pronounced increase of (220) direction for $r_{\mathrm{H}}=75 \%$ may be due to some texturing of the partially crystallized film before annealing, where (220) preferentially oriented nanograins are already formed.

In summary, for all the samples, the thermal annealing determines a strong crystallization of the layers, which shows after annealing a crystalline fraction of about $70 \%-$ $75 \%$. The characteristics of the crystallized layers strongly depend on the structure of the as-deposited ones:

for the layers which are fully amorphous before annealing, homogeneous crystallization takes place, leading to randomly oriented nanocrystalline layers. In this regime $\left(r_{\mathrm{H}} \leqslant 20 \%\right)$, there is a strong correlation between the hydrogen content before annealing, the residual stress in the crystals and the optical band gap, as already discussed.

(ii) for the layers which show some crystalline fraction already before annealing $\left(r_{\mathrm{H}} \geqslant 50 \%\right)$, the preferential growth of (220) nanocrystals already existing before annealing leads to a more textured structure.

However, in both cases no significant differences are observed in the final crystalline fraction. The presence of a residual amorphous contribution in the annealed layers-of about 25\%-30\%, according to the data shown in Table Iagrees with the previous observations from Harbeke et al. ${ }^{38}$ on thermally recrystallized $\mathrm{Si}$ layers deposited by lowpressure CVD technique. This amorphous contribution has been assumed to be mainly localized in the grain boundary regions. In relation to nanocrystalline Si layers obtained by other techniques as chemical transport, ${ }^{28}$ we observe a significant decrease in the residual stress in the Si crystals, which, as already indicated, has been related to the critical role of hydrogen in the relaxation of the structure during annealing. Finally, the partial texturing observed in the layers which showed some crystalline contribution already before annealing, agrees with the results obtained by other authors as Sun et al. ${ }^{39}$ who have observed the prevalence of the (220) growth for nanocrystalline samples deposited by sputtering in an hydrogen plasma at $T_{S}=250{ }^{\circ} \mathrm{C}$ (as in our case). This agreement confirms the critical dependence of the structure of the annealed layers on the presence in the as- 
deposited ones of some crystalline contribution, which is always observed in the literature when the layers are deposited with a high content of hydrogen in the plasma.

\section{CONCLUSION}

In summary, this work provides a significant contribution to the study of the impact of prior presence of hydrogen on the microstructure and properties of the thermally nanocrystallized silicon films. It was found that the network is more relaxed, i.e., the stress $\sigma$ is minimum, when the hydrogen content, $C_{\mathrm{H}}$, in the basic layer is maximum. This was found to correspond also to the widening of the optical band gap, $E_{\mathrm{og}}$. Both $\sigma$ and $E_{\mathrm{og}}$ clearly correlate with $C_{\mathrm{H}}$ which is related to the reactional mechanism of $\mathrm{Si}-\mathrm{H}_{2}$ that has been identified by infrared technique.

These features are less evident for samples already partially crystallized $\left(r_{\mathrm{H}} \geqslant 50 \%\right)$. The presence of a relatively high density of crystallites in the as-deposited layer $\left(r_{\mathrm{H}}\right.$ $=75 \%$ ) has led to an important texturing of the thermally crystallized samples, with a substantial increase of (220) direction at the expense of (111), as well as a decrease of the stress.

\section{ACKNOWLEDGMENTS}

The authors are indebted to P. Voivenel for his technical assistance and also to E. Balanzat for making possible the infrared measurements. This work has been partially funded by the French-Spanish "Actions Intégrées-Picasso" Cooperation programme (Project Ref. 96091) and the Spanish CICYT under Project Ref. MAT96-1010-C02-01.

${ }^{1}$ Polycrystalline Semiconductors IV_Physics, Chemistry and Technology, edited by S. Pizzini, H. P. Strunk, and J. H. Werner (Transtec., Zürich, 1996).

${ }^{2} \mathrm{C}$. Wang and G. Lucovsky, Proceedings of the 21th IEEE PVSC (IEEE, New York, 1990), p. 1614.

${ }^{3}$ S. D. Brotherton, D. J. McCulloch, J. B. Clegg, and J. P. Gowers, IEEE Trans. Electron Devices 40, 407 (1993).

${ }^{4}$ A. Borghesi, M. E. Giardini, M. Marazzi, A. Sassella, and G. de Santi, Appl. Phys. Lett. 70, 892 (1997).

${ }^{5}$ K. Tanaka, Mater. Res. Soc. Symp. Proc. 452, 3 (1997).

${ }^{6}$ A. Achiq, R. Rizk, R. Madelon, F. Gourbilleau, and P. Voivenel, Thin Solid Films 296, 15 (1997).

${ }^{7}$ T. Akasaka and I. Shimizu, Appl. Phys. Lett. 66, 3441 (1995).

${ }^{8}$ W. C. Sinke, T. Warabisako, M. Miyao, T. Tokayama, S. Roorda, and F. W. Saris, J. Non-Cryst. Solids 99, 308 (1988).

${ }^{9}$ A. A. Langford, M. L. Fleet, A. P. Nelson, W. A. Landford, and N. Maley, Phys. Rev. B 45, 13367 (1992).
${ }^{10}$ M. H. Brodsky, M. Cardona, and J. J. Cuomo, Phys. Rev. B 16, 3556 (1977).

${ }^{11}$ H. Shanks, C. J. Fang, L. Ley, M. Cardona, F. J. Demond, and S. Kalbitzer, Phys. Status Solidi B 100, 43 (1980).

${ }^{12} \mathrm{~W}$. Bayer, in Tetrahedrally Bonded Amorphous Semiconductors, edited by D. Adler and H. Fritzsche (Plenum, New York, 1985), p. 129.

${ }^{13}$ D. Das, Phys. Rev. B 51, 10729 (1995).

${ }^{14}$ P. M. Fauchet and I. H. Campbell, Crit. Rev. Solid State Mater. Sci. 14, 579 (1988).

${ }^{15}$ M. Yang, D. Huang, P. Hao, F. Zhang, X. Hou, and X. Wang, J. Appl. Phys. 75, 651 (1994).

${ }^{16}$ J. Macía, E. Martín, A. Pérez-Rodríguez, J. Jiménez, J. R. Morante, B. Aspar, and J. Magail, J. Appl. Phys. 82, 3730 (1997).

${ }^{17}$ H. Xia, Y. L. He, L. C. Wang, W. Zang, X. N. Liu, X. K. Zhang, and D. Feng, J. Appl. Phys. 78, 6705 (1995).

${ }^{18}$ Z. Sui, P. P. Leong, I. P. Herman, G. S. Higashi, and H. Temkin, Appl. Phys. Lett. 60, 2086 (1992).

${ }^{19}$ F. Anastassakis, in Physical Problems in Microelectronics, edited by J. Kassavod, Proceedings of the 4th International School of Physical Problems in Microelectronics (Varna, Bulgaria, 1985), p. 128.

${ }^{20}$ F. Bustarret, M. A. Hachida, and M. Brunel, Appl. Phys. Lett. 52, 1676 (1988).

${ }^{21}$ J. Tauc, R. Grigorovici, and A. Vancu, Phys. Status Solidi 15, 627 (1966).

${ }^{22}$ S. Oguz, D. A. Anderson, W. Paul, and H. J. Stein, Phys. Rev. B 22, 88 (1980).

${ }^{23}$ L. Lusson, A. Lusson, P. Elkaim, J. Dixmier, and D. Ballutaud, J. Appl. Phys. 81, 3073 (1997).

${ }^{24}$ Y. Mishima, M. Takei, T. Uematsa, N. Kakehi, U. Wakino, and M. Okabe, J. Appl. Phys. 78, 217 (1995).

${ }^{25}$ S. Boultadakis, S. Logothetidis, S. Ves, and J. Kircher, J. Appl. Phys. 73, 914 (1993).

${ }^{26}$ S. Veprek, Mater. Res. Soc. Symp. Proc. 164, 39 (1990).

${ }^{27}$ Y. Sun, R. Nishitani, and T. Miyasato, Jpn. J. Appl. Phys., Part 2 33, L1645 (1994).

${ }^{28}$ Z. Iqbal, F. A. Sarrott, and S. Veprek, J. Phys. C 16, 2005 (1983), and references therein.

${ }^{29}$ Dae Gyu Moon, Byung Hoo Jung, Jeong No Lee, Byung Tae Ahn, Ho Bin Im, Kee Soo Nam, and Sang Won Kang, J. Mater. Sci.: Mater. in Electron 5, 364 (1994).

${ }^{30}$ P. Hari, P. C. Taylor, and F. Finger, Mater. Res. Soc. Symp. Proc. 452, 791 (1997).

${ }^{31}$ J. Gonzalez-Hernandez, G. H. Azarbayejani, R. Tsu, and F. H. Pollak, Appl. Phys. Lett. 47, 1350 (1985).

${ }^{32}$ G. D. Cody, T. Tiedje, B. Abeles, B. Brooks, and Y. Goldstein, Phys. Rev. Lett. 47, 1480 (1981).

${ }^{33}$ A. Achiq, R. Rizk, R. Madelon, F. Gourbilleau, and P. Voivenel (unpublished).

${ }^{34}$ F. Urbach, Phys. Rev. 92, 1324 (1924).

${ }^{35}$ O. Schoenfeld, T. Hempel, X. Zhao, T. Sugano, and T. Aoyagi, Jpn. J. Appl. Phys., Part 1 33, 6082 (1994).

${ }^{36}$ C. C. Dollins, Acta Metall. 18, 1209 (1980).

${ }^{37}$ T. Kaneko, M. Wakagi, K.-I. Onisawa, and T. Minemura, Appl. Phys. Lett. 64, 1865 (1994)

${ }^{38}$ G. Harbeke, L. Krausbauer, E. F. Steigmeir, A. E. Widmer, H. F. Rappert, and G. Neugebauer, J. Electrochem. Soc. 131, 675 (1984).

${ }^{39}$ Y. Sun, T. Miyasato, and J. K. Wigmore, Appl. Phys. Lett. 70, 508 (1997). 